\title{
Narrativa
}

narrativa

Nuova serie

42 | 2020

Letteratura e economia nell'Italia degli anni Duemila

\section{La cura molteplice}

\author{
Laura Marzi
}

\section{OpenEdition}

\section{Journals}

Edizione digitale

URL: https://journals.openedition.org/narrativa/336

DOI: $10.4000 /$ narrativa.336

ISSN: 2804-1224

\section{Editore}

Presses universitaires de Paris Nanterre

\section{Edizione cartacea}

Data di pubblicazione: 1 décembre 2020

Paginazione: 107-117

ISBN: 978-2-84016-381-7

ISSN: $1166-3243$

Notizia bibliografica digitale

Laura Marzi, «La cura molteplice», Narrativa [Online], 42 | 2020, online dal 01 décembre 2021 consultato il 08 décembre 2021. URL: http://journals.openedition.org/narrativa/336 ; DOI: https:// doi.org/10.4000/narrativa.336

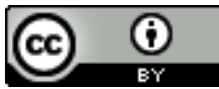

Narrativa est mise à disposition selon les termes de la Licence Creative Commons Attribution 4.0 International. 


\section{La cura molteplice}

\section{RiASSUNTO}

La condizione delle lavoratrici di cura può essere definita attraverso il concetto di invisibilità proposto da Axel Honneth (2001), come una forma acuta di discriminazione che interessa alcuni gruppi, relegati così alla non esistenza sociale. Il testo letterario effettua una inversione rispetto a tale condizione di invisibilità delle care-givers, ponendole, in quanto personaggi protagonisti, al centro della scena.

\section{RÉSUMÉ}

À partir de la réflexion sur l'invisibilité d'Axel Honneth (200)1, la condition des care givers peut être définie en tant que forme aigue de discrimination qui concerne certains groupes sociaux, ainsi condamnés à l'invisibilité sociale. Le texte littéraire peut mettre fin à cette invisibilité en donnant aux care givers le rôle central de protagonistes.

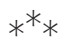

Marie Garrau e Alice Le Goff notano che il care (in italiano utilizziamo il termine cura) è un'attitudine relazionale che prevede attenzione, preoccupazione, sollecitudine: sono tutti aspetti della cura ${ }^{1}$.

Gli studi femministi e di genere ne hanno fatto emergere l'importanza politica e sociale: siamo state tutte e tutti in qualche modo destinatari di attività quotidiane e ordinarie di cura, eppure essa è stata esclusa dalla riflessione politica a causa del suo legame con la sfera domestica.

1. Garrau Marie, Le Goff Alice, Care, justice et dépendance. Introduction aux théories du Care, Paris, PUF, 2010, p. 5. 
Negli anni Settanta le femministe hanno spinto a riconsiderare i meccanismi della produzione a partire dai tempi di lavoro gratuito effettuato dalle donne a casa: il lavoro domestico. Esso si definisce come: "insieme di attività legate alla cura delle persone svolte nel contesto familiare - matrimoniale o familiare lavoro gratuito effettuato essenzialmente dalle donne"2.

La cura adesso, però, è anche un dispositivo critico che tocca prospettive disciplinari molto diverse: dalla filosofia morale fino alla psicologia dello sviluppo e del lavoro, la sociologia, gli studi in scienze infermieristiche e in medicina, ma anche la letteratura. Perché la cura suscita una tale attenzione in contesti così diversi?

La sua importanza è sicuramente connessa ai cambiamenti demografici che riguardano la società contemporanea, come l'invecchiamento della popolazione, le trasformazioni familiari (separazioni, madri single, famiglie omosessuali) e che hanno contribuito ad aumentare il bisogno di lavoro di cura. In particolare, il numero sempre crescente di donne che lavorano fuori casa e che non possono farsi interamente carico del lavoro domestico e/o della cura dei genitori anziani o di parenti disabili.

Anche per questo è importante comprendere l'urgenza di una riflessione politica sulla cura. Il merito delle teorie femministe sulla cura risiede nella prospettiva che forniscono per leggere questi fenomeni che riguardano la società contemporanea: la prospettiva della vulnerabilità e della dipendenza. In effetti focalizzarsi sulla vulnerabilità e sulla dipendenza universali ci ricorda che siamo esseri viventi con un corpo che ha dei limiti; ci riconduce alla nostra condizione contingente, di persone esposte agli altri.

\section{LA PROSPETTICA ETICA DEL CARE}

Il concetto di care è stato introdotto da Carol Gilligan ${ }^{3}$ a seguito di ricerche empiriche condotte in risposta alla teoria di Lawrence Kohlberg ${ }^{4}$ sulla capacità di giudizio morale. A quell'epoca in effetti Gilligan collaborava con Kohlberg all'università di Harvard. Il filosofo, nella sua teoria dello sviluppo morale, aveva

2. Hirata Helena, Laborie Françoise, Le Doare Hélène, Senotier Danièle, Dictionnaire critique du féminisme, Paris, PUF, 2000, p. 249. Le traduzioni, quando non diversamente segnalato, sono mie.

3. Gilligan Carol, In a Different Voice: Psychological Theory and Women's Development, Harvard, Harvard University Press, 1982.

4. Kohlberg Lawrence, The philosophy of moral development, San Francisco, Harper and Row, 1981. 
rilevato una deficienza nelle risposte delle donne, considerando come grado maggiore di moralità la messa in atto di principi di giustizia astratti e imparziali. Nella sua ricerca Gilligan ha semplicemente spostato l'ipotesi di una inadeguatezza delle donne sulla deficienza della teoria:

Nel momento in cui scrivevo il libro avevo preso coscienza di un problema inerente alla teoria psicologica che era in parte un problema metodologico (l'esclusione delle donne e delle persone di colore dal campo di studi sullo sviluppo morale) e in parte un problema teorico (il valore attribuito all'autonomia e alla razionalità divenuti criteri per stabilire il grado di sviluppo morale $)^{5}$.

Il problema, in effetti, dal punto di vista metodologico era piuttosto evidente: lo studio di Kohlberg a cui Gilligan lavorava non prendeva in considerazione il punto di vista delle donne: non venivano intervistate. Kohlberg fonda la sua teoria sull'osservazione di 84 ragazzi, mentre quando per la sua analisi comincerà a considerare le donne, concluderà che non sono in grado di superare il terzo dei sei stadi di sviluppo morale che egli ha individuato. Il filosofo decreterà infatti che le loro risposte evidenziano una difficoltà a formulare giudizi morali astratti e imparziali fatti in nome della comprensione razionale dell'esistenza di diritti umani universali.

Gilligan dimostrerà che Kohlberg non riconosce il valore della prospettiva che le donne intervistate propongono e le esigenze alle quali danno voce nel loro processo di formulazione di un giudizio morale. Lo studioso statunitense le relegherà semplicemente tra le prove di una incapacità.

Cosa emerge dalle risposte delle donne intervistate?

Gilligan riassume la ragione del giudizio di deficienza morale delle donne dato da Kohlberg nel fatto che secondo lo studioso "la preoccupazione per l'altro e per i rapporti umani risulta piuttosto come una debolezza che come una forza", come si verifica nel caso di Amy. La risposta di questa ragazzina di undici anni, intervistata nell'ambito di una ricerca di Kohlberg a cui lavorava anche Gilligan, sarà proprio il punto di partenza del testo di Gilligan In a different voice, l'opera considerata fondativa degli studi sul care.

Amy è intervistata con un ragazzo della sua età: Jake. Ai due viene proposto lo stesso dilemma detto di Heinz: Heinz, la cui moglie è malata, non ha il denaro

5. GiLligan Carol, Un regard prospectif à partir du passé, in Nurock Vanessa (a cura di), Carol Gilligan et l'éthique du care, Paris, PUF, 2010, p. 19.

6. Gilligan Carol, Une voix différente. Pour une éthique du care, Paris, Flammarion, 2008, p. 37. 
per comprare i farmaci necessari. Si trova allora di fronte alla seguente alternativa: rubarli o lasciare morire sua moglie. Ecco le risposte date dai due ragazzini: Jake considera senza nessuna esitazione che Heinz deve rubare i farmaci; Amy suggerisce invece che ci sono altre soluzioni. Che cosa succederà a sua moglie se Heinz andrà in prigione per il furto? Chi si occuperà di lei? Amy propone che Heinz parli al farmacista e che trovino insieme una soluzione.

Nella sua risposta Amy si concentra sul rifiuto del farmacista di rispondere al bisogno di Heinz, mentre Jake si focalizza sulla scelta tra il rispetto della legge e il diritto alla vita. Amy secondo Gilligan "non concepisce il dilemma come un problema matematico, ma come una narrazione di rapporti umani i cui effetti si prolungano nel tempo" ". Nella teoria degli stadi di Kohlberg, Jake riesce a raggiungere un livello tra il terzo e il quarto. Secondo l'interpretazione dello studioso statunitense, il ragazzo utilizza la logica con abilità, è capace di distinguere tra la morale e la legge ed è sicuro di avere effettuato una scelta corretta. La risposta di Amy, al contrario, si posiziona a uno stadio inferiore: secondo Kohlberg manifesterebbe un senso di inferiorità rispetto al mondo e un'incapacità di individuare le connessioni tra la morale e la legge.

La reazione di Gilligan è semplicemente di non fermarsi all'ipotesi di una inferiorità morale della ragazzina, ma di leggere la sua reazione per ciò che dice: nella risposta di Amy risiede la prospettiva etica del care. La ragazzina dà una risposta al dilemma considerando la posizione di Heinz come soggetto situato innanzitutto in un contesto relazionale e specifico. Le due concezioni, quella espressa da Jake, definita della giustizia, cioè della scelta morale fatta in nome di diritti e principi universali, e quella del care non sono affatto dicotomiche. Si tratta di fare, nei diversi contesti, una scelta rispetto alla prospettiva etica da prediligere.

\section{L'INVISIBILITÀ DEL LAVORO DI CURA}

L'invisibilità del lavoro di cura ha diverse cause. In primo luogo: "il lavoro di cura deve scomparire, dalla sua invisibilità dipende il suo successo"s. La buona riuscita di questo lavoro dipende infatti dalla capacità di chi lo svolge di renderlo e di rendersi invisibile: una buona assistente personale non può passare il suo tempo a ripetere quanto la persona anziana o disabile di cui si occupa sia incapace di svolgere questa o quella attività quotidiana, che ricade quindi su di lei.

\section{Ibid., p. 53.}

8. Molinier Pascale, "Le care à l'épreuve du travail", in Paperman Patricia, Laugier Sandra (a cura di), Le Souci des autres, cit., p. 343. 
Agirà in modo da occuparsene, cercando il più possibile di non sottolineare l'incapacità del suo assistito. Nel caso di lavoro di cura inteso come lavoro domestico, Molinier sottolinea ugualmente l'importanza della discrezione della lavoratrice o del lavoratore che deve essere capace di "fare le pulizie senza alterare l'ordine della scrivania dell'intellettuale"'.

In secondo luogo, la condizione di coloro che svolgono il lavoro di cura può essere definita attraverso l'elaborazione di Axel Honneth sul concetto di invisibilità ${ }^{10}$. Il filosofo tedesco, a partire dall'analisi del romanzo di Ralph Ellison L'uomo invisibile, descrive l'invisibilità come una forma acuta di discriminazione che riguarda alcuni gruppi sociali, relegati alla non esistenza sociale. Tra gli esempi di questa discriminazione attuata attraverso la cancellazione dal campo visivo, Honneth cita quello della lavoratrice domestica "a causa del suo statuto sociale considerato insignificante" ${ }^{11}$ e aggiunge:

Che si tratti di vedere la signora delle pulizie o la persona nera umiliata, sono in entrambi i casi oggetti distinti e facilmente identificabili nel campo visivo; di conseguenza l'invisibilità qui non può essere collegata a un fatto cognitivo, ma a una situazione sociale specifica ${ }^{12}$.

Audre Lorde scrive: "black women $[\ldots]$ have been rendered invisible through the depersonalization of racism"13 mentre Avishai Margalit nota che ignorare le persone non corrisponde a un atto di reificazione ma appunto a un accecamento volontario che ha come obbiettivo quello di cancellare alcune categorie sociali ${ }^{14}$.

Nel caso di coloro che svolgono il lavoro di cura, questa invisibilità rinvia a ciò che Joan Tronto, riferendosi alla condizione sociale di questi operatori, definisce "l'indifferenza dei privilegiati" Pascale Molinier e Patricia Paperman nel seguente modo: "le classi intellettuali

9. Ibid., p. 342.

10. Honneth Axel, "Invisibilité: sur l'épistémologie de la 'reconnaissance", Réseaux, n. 129, 2005, pp. 9-57.

11. Ibid., p. 42.

12. Ibid.

13. Lorde Audre, The trasformation of silence into Language and Action, in LORDE Audre, Sister Outsider. Essays and Speeches, Freedom, The Crossing Press Feminist Series, 1984, p. 42.

14. Margalit Avishai, La société décente, Paris, Flammarion, 2007, p. 101.

15. Hochschild Arlie Russel, Gilligan Carol, Tronto Joan C., Contre l'indifférence des privilégiés. A quoi sert le care, Paris, Payot, 2013. 
e privilegiate rifiutano di immaginare un'etica della cura che emani dalle persone che sono loro subalterne e meno colte" 1 .

Definendo l'invisibilità a partire dal suo contrario, cioè la visibilità, Honneth descrive la capacità visiva come una prima forma di conoscenza "la visibilità implica una forma elementare d'identificazione individuale e rappresenta di conseguenza una prima forma, primitiva, di ciò che definiamo conoscere" ${ }^{17}$. Vedere qualcosa costituisce una prima forma di conoscenza di quella cosa: al contrario, l'accecamento volontario presuppone la scelta di non conoscere, di ignorare, di restare indifferenti.

Questo legame di cui scrive Honneth tra la conoscenza e la visibilità è anche all'origine dell'uso quotidiano delle espressioni: "je vois" (io vedo) per dire: "capisco", che si ritrova anche nella lingua inglese (I see/I Know) come sottolinea il linguista Wolfang Bublitz nella sua analisi della metafora della vista ${ }^{18}$.

L'uso del verbo "vedere" come sinonimo del verbo "comprendere" presuppone esattamente ciò che scrive Honneth: l'azione di vedere è una prima forma di conoscenza. Si tratta di una sovrapposizione presente anche nel greco antico dove in effetti oî́ $\alpha$ è l'aoristo del verbo ógó $\omega$ (io vedo) e si traduce "io vidi", ma oî̉ $\alpha$ è anche una forma verbale indipendente con una flessione completa che significa: "io so". La ragione è la seguente: se nel passato io vidi, nel presente so. Di conseguenza se non vedo, non conoscerò mai.

L'invisibilità della cura, delle signore delle pulizie, delle persone di colore di cui scrive Honneth, comporta una mancanza di conoscenza delle loro condizioni di vita e si trasforma in seguito in un atto di sopraffazione di cui uno degli aspetti è l'indifferenza dei privilegiati.

Teresa de Lauretis scrive: "what is finally at stake is not how much to make visible the invisible as how to produce the conditions of visibility for a different social subject" ${ }^{\prime 19}$. I testi letterari che raccontano storie di lavoratrici di cura fanno esattamente ciò che la filosofa italiana ritiene fondamentale: creano le condizioni di visibilità necessarie.

16. Paperman Patricia, Molinier Pascale, "Désenclaver le care?”, in Gilligan Carol, Hochschild Arlie, Tronto Joan,C., Contre l'indifférence des privilégiés, cit., pp. 10-11.

17. Honneth Axel, "Invisibilité: sur l'épistémologie de la 'reconnaissance", cit., p. 44.

18. Bublitz Wolfram, "Seeing as a metaphor of Understanding: the visible and the invisible", in Schuth Andreas J., Horner Kristine, Weber Jean Jacques, (a cura di), Life in language. Studies in honour of Wolfgang Küblwein, Trier, Wissenschaftlicher Verlag, 2005, pp. 135-149.

19. De Lauretis Teresa, Alice doesn't, Bloomington, Indiana University Press, 1982, p. 9. 
Prima di tutto perché leggere presuppone l'azione di vedere ${ }^{20}$. Inoltre la letteratura permette anche di "andare a vedere" mettendoci al centro degli scenari del lavoro di cura. Per esempio, nel suo "Care": prendersi cura. Un lavoro inestimabile ${ }^{21}$ Molinier ribadisce spesso che le lavoratrici di Villa Plénitude, il nome fittizio che ha dato alla casa di riposo dove ha svolto la sua ricerca sul campo, ripetevano che tutti dovevano vedere in che cosa consiste il loro lavoro: cosa significa lavorare con delle persone molto malate o molto anziane, dover fronteggiare la mancanza di comprensione delle loro famiglie e le difficoltà pratiche. Eppure, rispetto alle rivendicazioni delle lavoratrici che si sentono non capite, pur riconoscendo del tutto le loro ragioni, la psicologa francese sottolinea che il lavoro di cura, come si diceva, è "fatto per non essere visto"22.

\section{ROMANZI DI CURA: UN ORDINE SIMBOLICO REALISTA}

Grazie alla narrazione, il lavoro e le lavoratrici di cura diventano le protagoniste della scena, sono in primo piano. Quindi, i romanzi che raccontano le storie di vita di queste lavoratrici costituiscono una sfida rispetto all'indifferenza dei privilegiati. Infatti, i romanzi di cura non si limitano a creare le condizioni di visibilità per le lavoratrici di cura, invisibilizzate appunto da dinamiche discriminatorie di classe, razza e genere, essi pongono queste donne al centro della scena. Generano, così, uno spostamento nell'ordine simbolico, ponendo, come suggerisce de Lauretis, le condizioni di visibilità per dei soggetti subalterni.

Secondo Luisa Muraro l'ordine simbolico è come il tempo, cioè un'istanza mediatrice che rende pensabile il reale: "gli esseri umani (detti animali razionali, ma sarebbe più giusto dire animali simbolici) hanno bisogno della mediazione, per conoscere non solo ciò che è distante e assente, ma anche ciò che è vicino e presente" 23 .

L'ordine simbolico così descritto corrisponde a ciò che Gilles Deleuze definisce il muro dei significati ai quali siamo come spillati, in cui si iscrivono tutte le determinazioni oggettive che ci inquadrano, ci identificano e ci permettono di riconoscerci ${ }^{24}$. Successivamente Deleuze dichiara che la letteratura, in quanto

20. Nel caso dei testi per non vedenti, utilizzando il braille la lettura è attraverso il contatto.

21. Molinier Pascale, "Care": prendersi cura. Un lavoro inestimabile, Milano, Moretti e Vitali, 2019.

22. Ibid., p. 157.

23. Muraro Luisa, L'ordine simbolico della madre, Roma, Editori Riuniti, 1991, p. 50.

24. Deleuze Gilles, Parnet Claire, Dialogues, Paris, Flammarion, 1996. 
"ligne de fuite" è esattamente lo strumento che permette la decostruzione di quel muro. Nelle stesse pagine, Deleuze parla del "devenir femme" della letteratura, intendendo in questo modo la possibilità per $i$ testi letterari di fare diventare, dunque di permettere la realizzazione in quanto avvenimento, di movimenti differenti rispetto a quelli della maggioranza.

Secondo Deleuze e Muraro la narrazione offre gli strumenti per modificare l'immaginario, per alterarlo. Riprendendo l'immagine di Deleuze della distruzione (ébranlement), grazie alla letteratura, del muro dei significati a cui siamo inchiodati, i romanzi che raccontano storie di cura agiscono questo atto decostruttivo in vari modi.

Nella nostra società coloro che svolgono un lavoro di cura occupano le ultime posizioni della scala sociale, in quanto quello di cura è considerato un lavoro tendenzialmente indegno, perché riguarda la sfera del bisogno e domestica, il corpo vecchio e/o malato. Per questo, nella nostra società questo lavoro è svolto da donne povere e straniere che nei romanzi che le vedono protagoniste restano tali (povere, donne, straniere) ma protagoniste. Effettuando questo passaggio da soggetti invisibili a personagge $e^{25}$ principali, i romanzi di cura invertono la condizione di invisibilità che condiziona le care workers creando una piccola rivoluzione nell'ordine simbolico.

Ciò che è importante sottolineare è che i romanzi che raccontano storie di cura, nei quali quindi le lavoratrici sono protagoniste, riproducono situazioni comuni della vita italiana contemporanea che però restano per lo più nascoste.

Nel romanzo di Titti Marrone La donna capovolta, la badante Alina è una donna moldava che fa l'assistente personale perché ha bisogno di guadagnare: "sono scappata dal mio paese dieci anni fa per la fame e la miseria e adesso sono di nuovo in cerca di lavoro, qui in Italia" ${ }^{26}$. Ugualmente realista è l'attitudine che Eleonora, la datrice di lavoro, ha nei confronti di Alina:

L'ho trovata. Non mi voglio sbilanciare in entusiasmi eccessivi, ma forse ci siamo. Si chiama Alina. Viene dalla Moldavia, è un'anima lunga, una bionda slavata. Sottile ma di struttura solida, parla abbastanza bene l'italiano o più probabilmente finge di conoscerlo, ma se non altro sembra capire qualcosa. Né troppo vecchia né troppo giovane, niente grilli per la testa. $\mathrm{O}$ almeno così si spera: dice che ha famiglia nel suo paese, un marito, una nipote che si occupa di suo padre. Ha anche un figlio che studia da qualche parte in Europa. Lavora per

25. Mazzanti Roberta, Neonato Silvia, Sarasini Bia, L’invenzione delle personagge, Roma, Iacobelli Editore, 2016.

26. Marrone Titti, La donna capovolta, Roma, Iacobelli Editore, 2019, p. 13. 
mandare i soldi a loro. Documenti in ordine e una complicazione in meno: non c'è bisogno del permesso di soggiorno perché ha anche il passaporto romeno. Quindi risulta cittadina europea. Meglio, non è propriamente romena, che quelle sono spesso ladre e bugiarde. E non mi ispirano fiducia. Questa invece è nata e cresciuta in un piccolo Paese dove la gente ha imparato a vivere con poco e accontentarsi facilmente ${ }^{27}$.

Mentre nel passaggio precedente emergono la condizione di povertà e la necessità di emigrare di Alina, in questo sono in primo piano la diffidenza e la discriminazione razziale di cui spesso le care workers sono vittime.

Titti Marrone racconta l'invisibilità del lavoro di cura e di chi lo effettua attraverso le parole di Alina: "l'ho imparato fin dal primo lavoro in Italia, dieci anni fa: per resistere in questa vita di accudimento a sfinteri allentati, svuotamento di padelle e imbracamento di pannoloni, devo nascondermi e vivere come in apnea"28.

Giulia Corsalini nel suo romanzo La lettrice di Cechov ${ }^{29}$ racconta un'altra verità sulle lavoratrici di cura che molti conoscono, ma pochi ricordano: che le donne che arrivano nel nostro paese per occuparsi di anziani e disabili molto spesso approdano qui da ben altri percorsi professionali. Indimenticabile, a riguardo, la feroce sagacia dello scrittore premio Nobel John M. Coetzee che nel romanzo Slow $\mathrm{Man}^{30}$ fa emergere come Marijana, la donna balcanica che si occupa del protagonista rimasto senza una gamba, non sia una donna di campagna, abituata a lavorare in una fattoria occupandosi di animali e bambini, come crede invece Paul invaghitosi di lei, ma una restauratrice, nata e vissuta in città, prima di aver attraversato l'Oceano per cercare di garantire ai propri figli la possibilità di studiare.

La protagonista del romanzo di Corsalini è una studiosa di Cechov, che aveva sì rinunciato alla carriera accademica già prima di trasferirsi in Italia, ma è rimasta una donna che crede che "il lavoro intellettuale in sé e la sua comunicazione a una classe di studenti continuano a dare un senso al mondo che ho di fronte" 31 .

27. Ibid., p. 25

28. Ibid., p. 33.

29. Corsalini Giulia, La lettrice di Cechov, Milano, nottetempo, 2018.

30. Coetzee John, M., Slow Man, London, Penguin Books, 2005.

31. Corsalini Giulia, La lettrice di Cechov, cit., p. 35. 
Anche Alina d'altra parte è una donna che nasconde la sua abilità con l'italiano, il suo livello culturale, facendo credere a Eleonora, quando inizia a lavorare per lei, di avere una conoscenza della nostra lingua appena accettabile:

dovrò stare attenta a Eleonora. Non dico piacerle, che non è possibile. Essere risolutiva ma silenziosa. Evitare di strafare, errore in cui talvolta sono portata a incorrere. In sua presenza, trasformarmi in un ficus o altra pianta da interni, quando occorre. Parlare pochissimo, e non tanto bene, l'italiano. Bene abbastanza, perché lei possa pensare che io capisca e mi sappia destreggiare con doverosa obbedienza, però non troppo. Decentemente perché Eleonora possa dire orgogliosa di sé alle sue amiche di esser stata brava a trovare una che spiccica la lingua, ma senza esagerare. $\mathrm{E}$ - non sia mai - senza dire di essere stata ingegnere nel mio Paese. Sembrerebbe una vanteria e poi a loro non interess $a^{32}$.

Come si può notare da questi accenni alle tre personagge care workers, Alina, Marijana e Nina fanno esperienza di una invisibilità che non è solo quella politica analizzata nei saggi che studiano il lavoro di cura. Si tratta di un nascondimento di sé che non subiscono solo in quanto vittime del sistema neoliberista, ma che agiscono per adattarsi alla nuova condizione in cui si trovano nel momento in cui diventano delle "badanti". Si tratta di una prova evidente dello spostamento nell'ordine simbolico effettuato dalla prospettiva narrativa per cui le tre donne diventano protagoniste, cioè agiscono la loro storia. Nel caso specifico ciò comporta di assumere l'invisibilità che abbiamo visto essere costitutiva del care.

La narrazione letteraria della cura permette poi uno spostamento dal piano politico, fondamentale e notoriamente universale, tipico dei saggi che analizzano il care da diverse prospettive scientifiche, a quello personale. La filosofa Adriana Cavarero infatti definisce la letteratura il più antico gesto di cura nei confronti del particolare, del concreto, perché dà voce a delle storie che avvengono nelle cucine, nei corridoi delle scuole e degli ospedali ${ }^{33}$.

La conseguenza di questo cambiamento di prospettiva reso possibile dalla narrazione letteraria è che il discorso si sposta dall'invisibilità delle care workers mettendo in luce quella che definisco, a partire dal già citato concetto di Tronto di indifferenza, un'ignoranza dei privilegiati. Eleonora e la donna anziana di cui si occupa Nina nel romanzo di Corsalini, come Paul Rayment nel libro di Coetzee possono permettersi di ignorare la realtà delle vite delle persone che

32. Marrone Titti, La donna capovolta, cit., p. 33.

33. Cavarero Adriana, Tu che mi guardi, tu che mi racconti, Milano, Feltrinelli, 1997, p. 50. 
lavorano nelle loro case e si occupano dei loro cari. Secondo Caroline Ibos il fatto che i datori di lavoro delle care workers non si interessino affatto di conoscere la storia personale delle donne che si occupano dei loro figli o genitori anziani rappresenta, poi, un'inversione dello schema tradizionale sapere-potere ed è alla base dell'indifferenza rispetto ai diritti sociali di queste donne ${ }^{34}$.

I romanzi di cura, allora, non edulcorano lo scenario vigente. Al contrario, si potrebbe concludere che essi rappresentano un ordine simbolico realista: delle donne appartenenti a classi sociali subalterne che si occupano dei bisogni dei privilegiati e svolgono un ruolo fondamentale nella nostra società sono descritte attraverso una prospettiva veritiera: come protagoniste. Lo sono in effetti anche nella nostra società. Questo ordine realista rappresentato nei romanzi di cura, però, si oppone alla realtà sociale, che vuole ignorare queste donne e il loro lavoro, relegare la cura alla sfera domestica, per nascondere le ingiustizie sociali che il care sottende, ma anche per non mettere in discussione l'indipendenza dei privilegiati, mito fondativo della nostra cultura.

Laura MARZI

LEGS, Laboratoire d'études de genre et de sexualité

34. Iвоs Caroline, Qui gardera nos enfants?, Paris, Flammarion, 2012, p. 199. 